\title{
On Minimal Copulas under the Concordance Order
}

\author{
Jae Youn $A n^{1} \cdot$ Sebastian Fuchs ${ }^{2}$ \\ Received: 18 February 2019 / Accepted: 20 November 2019 / Published online: 10 December 2019 \\ (c) The Author(s) 2019
}

\begin{abstract}
In the present paper, we study extreme negative dependence focussing on the concordance order for copulas. With the absence of a least element for dimensions $d \geq 3$, the set of all minimal elements in the collection of all copulas turns out to be a natural and quite important extreme negative dependence concept. We investigate several sufficient conditions, and we provide a necessary condition for a copula to be minimal. The sufficient conditions are related to the extreme negative dependence concept of $d$-countermonotonicity and the necessary condition is related to the collection of all copulas minimizing multivariate Kendall's tau. The concept of minimal copulas has already been proved to be useful in various continuous and concordance order preserving optimization problems including variance minimization and the detection of lower bounds for certain measures of concordance. We substantiate this key role of minimal copulas by showing that every continuous and concordance order preserving functional on copulas is minimized by some minimal copula, and, in the case the continuous functional is even strictly concordance order preserving, it is minimized by minimal copulas only. Applying the above results, we may conclude that every minimizer of Spearman's rho is also a minimizer of Kendall's tau.
\end{abstract}

Keywords Concordance order - Countermonotonicity - Extreme negative dependence $\cdot$ Kendall's tau $\cdot$ Minimal copula $\cdot$ Optimization $\cdot$ Spearman's rho

Mathematics Subject Classification 49J99 - 49K99 - 60E05 - 60E15 - 62H20

Communicated by Francesco Zirilli.

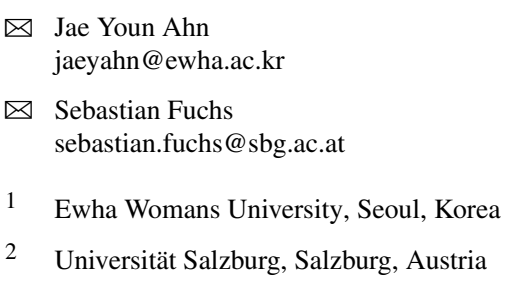




\section{Introduction}

The strongest notion of positive monotone association between random variables is given by comonotonicity. A $d$-dimensional continuous random vector is said to be comonotonic, if one of the following equivalent conditions is satisfied; see, e.g. [1]:

- the random vector has the upper Fréchet-Hoeffding bound $M$ as its copula,

- each of its coordinates is a.s. an increasing transformation of the other,

- each of its bivariate subvectors is comonotonic.

In many applications in finance and insurance, comonotonicity can be regarded as one of the most dangerous behaviours and, with its popularity in risk management as an extreme positive dependence, it seems quite reasonable that also extreme negative dependence is getting more attention; see, e.g. [2]. In the bivariate case, the strongest notion of negative monotone association between random variables is given by countermonotonicity. A bivariate continuous random vector is said to be countermonotonic, if one of the following equivalent conditions is satisfied; see, e.g. [1]:

- the random vector has the lower Fréchet-Hoeffding bound $W$ as its copula,

- each of its coordinates is a.s. a decreasing transformation of the other.

In contrast to comonotonicity, for dimensions $d \geq 3$, there exist no continuous random vector for which all the bivariate subvectors are countermonotonic and hence there exists no single agreed definition of extreme negative dependence in arbitrary dimension; see, e.g. [3-8].

In the present paper, we investigate extreme negative dependence focussing on the concordance order for copulas. It is well known that $M$ is the greatest element and, in the bivariate case, $W$ is the least element in the collection of all copulas with respect to concordance order. With the absence of a least element for dimensions $d \geq 3$, the set of all minimal elements (so-called minimal copulas) in the collection of all copulas turns out to be a natural and quite important extreme negative dependence concept, in particular, with regard to the minimization of continuous and concordance order preserving optimization problems. This includes several measures of dependence like Kendall's tau and Spearman's rho, but also the variance of the sum of several given random variables as a map on copulas; see, e.g. [9]. While it is well known that every continuous and (strictly) concordance order preserving functional on copulas is (uniquely) maximized by $M$ and, in the bivariate case, (uniquely) minimized by $W$, less is known about minimization for dimensions $d \geq 3$.

Most recently, Ahn [10] and Lee et al. [11] have demonstrated the potential of minimal copulas in variance minimization when the marginals are uniform, elliptical or belong to the unimodal-symmetric location-scale family; we refer to [7,12] for further results on variance minimization. Moreover, Genest et al. [13] and Lee and Ahn [6] have provided minimal copulas minimizing certain measures of concordance. Further continuous and concordance order preserving functionals on copulas are discussed in $[9,14]$, but also in [15-18] where the necessary properties of the functional strictly depend on the function to be integrated; for more details on this topic we refer to [17] and the references therein.

In this paper, we first discuss the existence and list some important examples of copulas that are minimal with respect to concordance order. We then investigate several 
sufficient conditions (Proposition 3.1), and we provide a necessary condition (Theorem 3.1) for a copula to be minimal. The sufficient conditions are related to the extreme negative dependence concept of $K$-countermonotonicity introduced in [6,11], and the necessary condition is related to the collection of all copulas minimizing multivariate Kendall's tau. It turns out that every minimal copula minimizes Kendall's tau which is the main result of this paper. We further point out the key role of minimal copulas with regard to the minimization of continuous and (strictly) concordance order preserving optimization problems. It turns out that every continuous and concordance order preserving functional on copulas is minimized by some minimal copula and that, in the case the continuous functional is even strictly concordance order preserving, it is minimized by minimal copulas only (Theorem 4.1). Finally, we apply our results to Kendall's tau and Spearman's rho and show that every minimizer of Spearman's rho is also a minimizer of Kendall's tau (Corollary 5.2). Some auxiliary results are established in the Appendix.

\section{Preliminaries}

In this section, we fix some notation and recall some definitions and results on copulas, a group of transformations of copulas and the concordance order.

Let $\mathbb{I}:=[0,1]$ and let $d \geq 2$ be an integer which will be kept fix throughout this paper. We denote by $\mathbf{e}_{1}, \ldots, \mathbf{e}_{d}$ the unit vectors in $\mathbb{R}^{d}$, by $\mathbf{0}$ the vector in $\mathbb{R}^{d}$ with all coordinates being equal to 0 and by 1 the vector in $\mathbb{R}^{d}$ with all coordinates being equal to 1 . For $\mathbf{u}, \mathbf{v} \in \mathbb{R}^{d}$, we use the notation $\mathbf{u} \leq \mathbf{v}$ resp. $\mathbf{u}<\mathbf{v}$ in the usual sense such that $u_{k} \leq v_{k}$ resp. $u_{k}<v_{k}$ holds for every $k \in\{1, \ldots, d\}$.

Copulas For $K \subseteq\{1, \ldots, d\}$, we consider the map $\eta_{K}: \mathbb{I}^{d} \times \mathbb{I}^{d} \rightarrow \mathbb{I}^{d}$ given coordinatewise by

$$
\left(\eta_{K}(\mathbf{u}, \mathbf{v})\right)_{k}:= \begin{cases}u_{k}, & k \in\{1, \ldots, d\} \backslash K, \\ v_{k}, & k \in K\end{cases}
$$

and, for $k \in\{1, \ldots, d\}$, we put $\eta_{k}:=\eta_{\{k\}}$. A copula is a function $C: \mathbb{I}^{d} \rightarrow \mathbb{I}$ satisfying the following conditions:

(i) $\sum_{K \subseteq\{1, \ldots, d\}}(-1)^{d-|K|} C\left(\eta_{K}(\mathbf{u}, \mathbf{v})\right) \geq 0$ holds for all $\mathbf{u}, \mathbf{v} \in \mathbb{I}^{d}$ such that $\mathbf{u} \leq \mathbf{v}$.

(ii) $C\left(\boldsymbol{\eta}_{k}(\mathbf{u}, \mathbf{0})\right)=0$ holds for every $k \in\{1, \ldots, d\}$ and every $\mathbf{u} \in \mathbb{I}^{d}$.

(iii) $C\left(\boldsymbol{\eta}_{k}(\mathbf{1}, \mathbf{u})\right)=u_{k}$ holds for every $k \in\{1, \ldots, d\}$ and every $\mathbf{u} \in \mathbb{I}^{d}$.

This definition is in accordance with the literature; see, e.g. [1,19]. We denote the collection of all copulas by $\mathcal{C}$. The following copulas are of particular interest:

- The upper Fréchet-Hoeffding bound $M$ given by $M(\mathbf{u}):=\min \left\{u_{1}, \ldots, u_{d}\right\}$ is a copula and every copula $C$ satisfies $C(\mathbf{u}) \leq M(\mathbf{u})$ for every $\mathbf{u} \in \mathbb{I}^{d}$.

- The independence copula $\Pi$ given by $\Pi(\overline{\mathbf{u}}):=\prod_{k=1}^{d} u_{k}$ is a copula.

- The lower Fréchet-Hoeffding bound $W$ given by $W(\mathbf{u}):=\max \left\{\sum_{k=1}^{d} u_{k}+1-\right.$ $d, 0\}$ is a copula only for $d=2$, and every copula $C$ satisfies $W(\mathbf{u}) \leq C(\mathbf{u})$ for every $\mathbf{u} \in \mathbb{I}^{d}$. 
Since every copula $C$ has a unique extension to a distribution function $\mathbb{R}^{d} \rightarrow \mathbb{I}$, there exists a unique probability measure $Q^{C}: \mathcal{B}\left(\mathbb{I}^{d}\right) \rightarrow \mathbb{I}$ satisfying $Q^{C}[[\mathbf{0}, \mathbf{u}]]=C(\mathbf{u})$ for every $\mathbf{u} \in \mathbb{I}^{d}$. The probability measure $Q^{C}$ is said to be the copula measure with respect to $C$ and it satisfies $Q^{C}[] \mathbf{u}, \mathbf{v}[]=Q^{C}[[\mathbf{u}, \mathbf{v}]]$ for all $\mathbf{u}, \mathbf{v} \in \mathbb{I}^{d}$ such that $\mathbf{u} \leq \mathbf{v}$.

A Group of Transformations of Copulas Let $\Phi$ denote the collection of all transformations $\mathcal{C} \rightarrow \mathcal{C}$ and consider the composition $\circ: \Phi \times \Phi \rightarrow \Phi$ given by $\left(\varphi_{2} \circ \varphi_{1}\right)(C):=\varphi_{2}\left(\varphi_{1}(C)\right)$ and the map $\iota \in \Phi$ given by $\iota(C):=C$. Then $(\Phi, \circ)$ is a semigroup with neutral element $\iota$. For $i, j, k \in\{1, \ldots, d\}$ with $i \neq j$, we define the maps $\pi_{i, j}, v_{k}: \mathcal{C} \rightarrow \mathcal{C}$ by letting

$$
\begin{aligned}
\left(\pi_{i, j}(C)\right)(\mathbf{u}) & :=C\left(\boldsymbol{\eta}_{\{i, j\}}\left(\mathbf{u}, u_{j} \mathbf{e}_{i}+u_{i} \mathbf{e}_{j}\right)\right) \\
\left(v_{k}(C)\right)(\mathbf{u}) & :=C\left(\boldsymbol{\eta}_{k}(\mathbf{u}, \mathbf{1})\right)-C\left(\boldsymbol{\eta}_{k}(\mathbf{u}, \mathbf{1}-\mathbf{u})\right)
\end{aligned}
$$

Each of these maps is an involution and there exists

- a smallest subgroup $\Gamma^{\pi}$ of $\Phi$ containing every $\pi_{i, j}$,

- a smallest subgroup $\Gamma^{v}$ of $\Phi$ containing every $v_{k}$ and

- a smallest subgroup $\Gamma$ of $\Phi$ containing $\Gamma^{\pi} \cup \Gamma^{\nu}$.

The transformations in $\Gamma^{\pi}$ are called permutations and the transformations in $\Gamma^{v}$ are called reflections. The group $\Gamma^{v}$ is commutative and for $K \subseteq\{1, \ldots, d\}$ we define $v_{K}:=\bigcirc_{k \in K} v_{k}$ (such that $v_{\emptyset}=\iota$ ). We note that the total reflection $\tau:=v_{\{1, \ldots, d\}}$ transforms every copula into its survival copula. From a probabilistic viewpoint, if $\mathbf{U}$ is a random vector whose distribution function is the copula $C$, then the reflected copula $v_{K}(C)$ of $C$ is the distribution function of the random vector $\eta_{K}(\mathbf{U}, \mathbf{1}-\mathbf{U})$. We refer to [20] for further details on the groups $\Gamma^{\pi}, \Gamma^{\nu}$ and $\Gamma$.

The Ordered Topological Space $\mathcal{C}$ Under the sup-norm, the collection $\mathcal{C}$ is a compact topological space.

A relation $\lessdot$ on $\mathcal{C}$ is said to be an order relation, if it is reflexive, antisymmetric and transitive; in this case, the pair $(\mathcal{C}, \lessdot)$ is called ordered set. The order relation $\lessdot$ is said to be closed, if the graph of $\lessdot$ is a closed set in $\mathcal{C} \times \mathcal{C}$. We denote by $m(\mathcal{C}, \lessdot)$ the set of all minimal elements of $(\mathcal{C}, \lessdot)$.

For $C, D \in \mathcal{C}$, we write $C \preceq D$, if $C(\mathbf{u}) \leq D(\mathbf{u})$ and $(\tau(C))(\mathbf{u}) \leq(\tau(D))(\mathbf{u})$ for all $\mathbf{u} \in \mathbb{I}^{d}$. Then $\preceq$ is a closed order relation which is called concordance order on $\mathcal{C}$.

Since $\tau(M)=M$, the upper Fréchet-Hoeffding bound $M$ is the greatest element in $(\mathcal{C}, \preceq)$; similarly, in the case $d=2$, the lower Fréchet-Hoeffding bound $W$ is the least element in $(\mathcal{C}, \preceq)$. A copula $C \in \mathcal{C}$ is said to be a minimal copula, if $C \in m(\mathcal{C}, \preceq)$.

Note that the ordered set $(\mathcal{C}, \preceq)$ is not a lattice: for $d=2$, this is immediate from [1, Theorem 7.3.5] and, for $d \geq 3$, the assertion follows using the same arguments as in the proof of [1, Theorem 7.3.5]. In this context, recall that, for $d=2$, concordance order and pointwise order coincide.

\section{Multivariate Countermonotonicity}

With the absence of a least element for dimensions $d \geq 3$, the set of all minimal elements, i.e. all locally least elements, in the collection of all copulas turns out to 
play an important role when studying extreme negative dependence concepts. In this section, we discuss the existence and list some important examples of copulas that are minimal with respect to concordance order (so-called minimal copulas). Additionally, we investigate several sufficient conditions and we provide a necessary condition for a copula to be minimal.

First sufficient and necessary conditions for a copula to be minimal can be achieved by comparing concordance order with other order relations:

Remark 3.1 Let $\lessdot$ be an order relation on $\mathcal{C}$.

(1) If $C \preceq D$ implies $C \lessdot D$, then $m(\mathcal{C}, \lessdot) \subseteq m(\mathcal{C}, \preceq)$.

(2) If $C \lessdot D$ implies $C \preceq D$, then $m(\mathcal{C}, \preceq) \subseteq m(\mathcal{C}, \lessdot)$.

Note that (1) is applicable to pointwise order and (2) is applicable to supermodular order; for more details on the comparison of concordance order with other order relations, we refer to [21-23].

We are now interested in sufficient and necessary conditions for a copula to be minimal that are formulated in terms of the copula itself.

A copula $C \in \mathcal{C}$ is said to be $K$-countermonotonic $(K-\mathrm{CM})$, if there exists

- some $K \subseteq\{1, \ldots, d\}$ with $2 \leq|K| \leq d$,

- a family $\left\{g_{k}\right\}_{k \in K}$ of strictly increasing and continuous functions $\mathbb{I} \rightarrow \mathbb{R}$ and

- some $c \in \mathbb{R}$

such that

$$
Q^{C}\left[\left\{\mathbf{u} \in \mathbb{I}^{d}: \sum_{k \in K} g_{k}\left(u_{k}\right)=c\right\}\right]=1
$$

compare [11, Definitions $2,3 \& 4$ ]. We denote by $\mathcal{C}_{K-\mathrm{CM}}$ the collection of all copulas that are $K$-CM. To ease notation, we write $d$-CM in the case $K=\{1, \ldots, d\}$. Note that, for $d=2$, a copula $C$ is 2-CM if and only if $C=W$. Thus, $K$-countermonotonicity may be regarded as a natural extension of countermonotonicity to dimensions $d \geq 3$.

For some particular choices $K \subseteq\{1, \ldots, d\}, K$-countermonotonicity implies minimality:

Proposition 3.1 The inclusion

$$
\mathcal{C}_{K-\mathrm{CM}} \subseteq m(\mathcal{C}, \preceq)
$$

holds for every $K \subseteq\{1, \ldots, d\}$ such that $2 \leq|K| \in\{d-1, d\}$. Moreover, $\mathcal{C}_{d-\mathrm{CM}}=$ $m(\mathcal{C}, \preceq)$ if and only if $d=2$; in this case $m(\mathcal{C}, \preceq)=\{W\}$.

Proof The inclusions were proved in [11, Theorem 4, Lemma 5], and the equivalence for $d=2$ follows from Example 3.2 (1) below.

Examples 3.2 below show that

(1) the inclusion in Proposition 3.1 for $|K|=d$ is strict whenever $d \geq 3$, 
(2) the inclusion in Proposition 3.1 for $|K|=d-1$ is strict, and that

(3) the inclusion in Proposition 3.1 fails to be satisfied whenever $2 \leq|K| \leq d-2$.

In the following, we list some important minimal copulas and show in passing that the set $m(\mathcal{C}, \preceq)$ is non-empty:

Example 3.1 (1) For every $K \subseteq\{1, \ldots, d\}$ with $1 \leq|K| \leq d-1$, the copula $v_{K}(M)$ satisfying $\left(v_{K}(M)\right)(\mathbf{u})=\max \left\{\min \left\{u_{k} \mid k \notin K\right\}-\max \left\{1-u_{k} \mid k \in K\right\}, 0\right\}$ (see, e.g. [24, Example 7.1]) is $d$-CM and hence minimal.

Indeed, consider $K \subseteq\{1, \ldots, d\}$ with $1 \leq|K| \leq d-1$. Then

$$
Q^{v_{K}(M)}\left[\left\{\mathbf{u} \in \mathbb{I}^{d}: \eta_{K}(\mathbf{u}, \mathbf{1}-\mathbf{u})=\alpha \mathbf{1} \text { for some } \alpha \in \mathbb{I}\right\}\right]=1
$$

By choosing $g_{k}\left(u_{k}\right)=u_{k} /|K|$ for every $k \in K$ and $g_{k}\left(u_{k}\right)=u_{k} /(d-|K|)$ for every $k \in\{1, \ldots, d\} \backslash K$, we obtain

$$
\begin{aligned}
\sum_{k=1}^{d} g_{k}\left(u_{k}\right) & =\sum_{k \in K} \frac{u_{k}}{|K|}+\sum_{k \in\{1, \ldots, d\} \backslash K} \frac{u_{k}}{d-|K|} \\
& =\sum_{k \in K} \frac{1-\alpha}{|K|}+\sum_{k \in\{1, \ldots, d\} \backslash K} \frac{\alpha}{d-|K|}=1
\end{aligned}
$$

for every $\mathbf{u} \in \mathbb{I}^{d}$ satisfying $\boldsymbol{\eta}_{K}(\mathbf{u}, \mathbf{1}-\mathbf{u})=\alpha \mathbf{1}$ for some $\alpha \in \mathbb{I}$, and hence $Q^{v_{K}(M)}\left[\left\{\mathbf{u} \in \mathbb{I}^{d}: \sum_{k=1}^{d} g_{k}\left(u_{k}\right)=1\right\}\right]=1$. The assertion then follows from Proposition 3.1.

(2) The copula $C$ given by

$$
C(\mathbf{u}):=\max \left\{\sum_{k=1}^{d} u_{k}^{1 /(d-1)}-(d-1), 0\right\}^{d-1}
$$

is Archimedean and is called the Clayton copula with parameter $-1 /(d-1)$. It follows from [6, Theorem 4] and Proposition 3.1 that $C$ is $d-\mathrm{CM}$ and hence minimal.

(3) Consider $d=3$ and the probability measure $Q: \mathcal{B}\left(\mathbb{I}^{3}\right) \rightarrow \mathbb{I}$ whose probability mass is distributed uniformly on the edges of the equilateral triangle in $\mathbb{I}^{3}$ with vertices $(0,1 / 2,1),(1 / 2,1,0)$ and $(1,0,1 / 2)$. By [25, Example 7], its corresponding distribution function $C$ is a copula satisfying $Q\left[\left\{\mathbf{u} \in \mathbb{I}^{3}: \sum_{i=1}^{3} u_{i}=3 / 2\right\}\right]=1$ which implies that $C$ is 3 -countermonotonic. It hence follows from Proposition 3.1 that $C$ is minimal.

Example 3.2 (1) Consider $d \geq 3$, a $(d-1)$-dimensional, $(d-1)$-CM copula $C$ and define the map $D: \mathbb{I}^{d-1} \times \mathbb{I} \rightarrow \mathbb{R}$ by letting $D(\mathbf{u}, v):=C(\mathbf{u}) v$. Then, by [26, Theorem 6.6.3], $D$ is a $d$-dimensional copula and it follows from Proposition 3.1 that $D$ is minimal. However, $D$ fails to be $d$-CM which follows from straightforward calculation. 
(2) Consider $d \geq 3$. Then the copula $C$ discussed in Example 3.1 (2) is $d$-CM and hence minimal. However, it is evident that $C$ fails to be $K$-CM whenever $2 \leq$ $|K| \leq d-1$.

(3) Consider $d \geq 4, K \subseteq\{1, \ldots, d\}$ with $2 \leq|K| \leq d-2$, a $|K|$-dimensional, $|K|-C M$ copula $C$ and define the maps $D, E: \mathbb{I}^{|\bar{K}|} \times \mathbb{I}^{d-|K|} \rightarrow \mathbb{R}$ by letting $D(\mathbf{u}, \mathbf{v}):=C(\mathbf{u}) \Pi(\mathbf{v})$ and $E(\mathbf{u}, \mathbf{v}):=C(\mathbf{u}) M(\mathbf{v})$. Then, by [26, Theorem 6.6.3], $D$ and $E$ are $d$-dimensional copulas and, by definition, are $\{1, \ldots,|K|\}$-CM. However, $D \preceq E$ with $D \neq E$ which implies that $E$ is not minimal.

Remark 3.2 The collections $\mathcal{C}_{K-\mathrm{CM}}$ with $K \subseteq\{1, \ldots, d\}$ such that $2 \leq|K|$ are not directed. Indeed, consider $K, L \subseteq\{1, \ldots, d\}$ with $2 \leq|K|$ and $2 \leq|L|$. Then, due to Examples 3.2 (1) and (2), $K \subseteq L$ neither implies $\mathcal{C}_{K-\mathrm{CM}} \subseteq \mathcal{C}_{L-\mathrm{CM}}$ nor $\mathcal{C}_{L-\mathrm{CM}} \subseteq \mathcal{C}_{K-\mathrm{CM}}$

We proceed with the discussion of a necessary condition for a copula to be minimal.

A copula $C \in \mathcal{C}$ is said to be Kendall-countermonotonic (Kendall-CM), if the identity

$$
\min \{C(\mathbf{u}),(\tau(C))(\mathbf{1}-\mathbf{u})\}=0
$$

holds for every $\mathbf{u} \in] \mathbf{0}, \mathbf{1}\left[\right.$. We denote by $\mathcal{C}_{\text {Kendall-CM }}$ the collection of all copulas that are Kendall-CM. The term Kendall-countermonotonicity is motivated by the fact that a copula $C$ is Kendall-CM if and only if $C$ minimizes multivariate Kendall's tau; see [5]. Kendall's tau is a map $\kappa: \mathcal{C} \rightarrow \mathbb{R}$ given by

$$
\kappa(C):=\frac{2^{d}}{2^{d-1}-1}\left(\int_{\mathbb{I}^{d}} C(\mathbf{u}) \mathrm{d} Q^{C}(\mathbf{u})-\frac{1}{2^{d}}\right)
$$

and the definition of Kendall's tau is in accordance with [27]. The following characterization of Kendall-countermonotonicity is due to [5, Lemma 3.1, Theorem 3.4]:

Proposition 3.2 For a copula $C \in \mathcal{C}$ the following are equivalent:

(1) $C$ is Kendall-CM.

(2) $\tau(C)$ is Kendall-CM.

(3) Every $\mathbf{u} \in] \mathbf{0}, \mathbf{1}\left[\right.$ satisfies $Q^{C}[[\mathbf{0}, \mathbf{u}]]=0$ or $Q^{C}[[\mathbf{u}, \mathbf{1}]]=0$.

(4) $\int_{\mathbb{I}^{d}} C(\mathbf{u}) \mathrm{d} Q^{C}(\mathbf{u})=0$.

It follows from [5, Theorem 3.3] that, for $d=2$, a copula $C$ is Kendall-CM if and only if $C=W$. Thus, Kendall-countermonotonicity may be regarded as a natural extension of countermonotonicity to dimensions $d \geq 3$.

Remark 3.3 A subset $A \subseteq \mathbb{I}^{d}$ is said to be strictly comonotonic, if either $\mathbf{u}<\mathbf{v}$ or $\mathbf{v}<\mathbf{u}$ for all $\mathbf{u}, \mathbf{v} \in A$. Consider now a copula $C$ for which there exists some strictly comonotonic set $A \subseteq] \mathbf{0}, \mathbf{1}$ [ consisting of at least two points such that $A \subseteq \operatorname{supp} Q^{C}$, i.e. the support supp $Q^{C}$ of $Q^{C}$ contains some strictly comonotonic subset of ]0, $\mathbf{1}$ [. It then follows from Proposition 3.2 (3) that such a copula $C$ fails to be Kendallcountermonotonic. 
Thus, one may interpret Kendall-countermonotonicity as the one extreme negative dependence concept where it is inadmissible for a copula to have some strictly comonotonic support.

The following theorem is the main result of this paper; it states that every minimal copula is Kendall-CM and hence every minimal copula minimizes Kendall's tau.

Theorem 3.1 We have

$$
m(\mathcal{C}, \preceq) \subseteq \mathcal{C}_{\text {Kendall-CM }}
$$

Moreover, if $d=2$, then $m(\mathcal{C}, \preceq)=\mathcal{C}_{\text {Kendall }-C M}=\{W\}$.

Proof The inclusion is proved in the appendix (see Lemmas A.1, A.2 and A.3); there, for a copula $C \in \mathcal{C} \backslash \mathcal{C}_{\text {Kendall-CM }}$, we construct a copula $D \in \mathcal{C}$ satisfying $D \preceq C$ with $D \neq C$ which then implies that $C$ is not minimal. The identity for $d=2$ follows from [5, Theorem 3.3].

The following example shows that the inclusion in Theorem 3.1 is strict whenever $d \geq 4$ :

Example 3.3 For $d \geq 4$, consider the maps $C, D: \mathbb{I}^{2} \times \mathbb{I}^{d-2} \rightarrow \mathbb{I}$ given by $C(\mathbf{u}, \mathbf{v}):=$ $W(\mathbf{u}) \Pi(\mathbf{v})$ and $D(\mathbf{u}, \mathbf{v}):=W(\mathbf{u}) M(\mathbf{v})$. By, [26, Theorem 6.6.3], $C$ and $D$ are copulas, and it follows from [5, Remark 3.1.(3)] that $D$ is Kendall-CM. However, $D$ fails to be a minimal copula which is a consequence of $C \preceq D$ with $C \neq D$.

It is interesting to note that also every $K-\mathrm{CM}$ copula is Kendall-CM:

Proposition 3.3 The inclusion

$$
\mathcal{C}_{K-\mathrm{CM}} \subseteq \mathcal{C}_{\text {Kendall-CM }}
$$

holds for every $K \subseteq\{1, \ldots, d\}$ such that $2 \leq|K| \leq d$. Moreover, $\bigcup_{K \subseteq\{1, \ldots, d\}, 2 \leq|K| \leq d}$ $\mathcal{C}_{K-\mathrm{CM}}=\mathcal{C}_{\text {Kendall-CM }}$ if and only if $d=2$.

Proof The inclusions follow from [6, Theorem 6], and the equivalence for $d=2$ is a consequence of Example 3.4 below.

The following example shows that the inclusions in Proposition 3.3 are strict whenever $d \geq 3$ :

Example 3.4 For $d \geq 3$, consider the copula

$$
C(\mathbf{u}):=\frac{1}{2^{d}-2} \sum_{K \subseteq\{1, \ldots, d\}, 1 \leq|K| \leq d-1} v_{K}(M)
$$

Then, $C$ is Kendall-CM but fails to be $K-\mathrm{CM}$ whenever $2 \leq|K| \leq d$. The result is a $d$-dimensional analogue of [5, Example 3.2]. 
In the following, we summarize the obtained inclusions:

$$
\begin{aligned}
& m(\mathcal{C}, \preceq) \quad \subseteq_{\mathrm{II}} \quad \mathcal{C}_{\text {Kendall-CM }} \\
& \mathrm{UI}_{\mathrm{I}} \quad \mathrm{UI}_{\mathrm{IV}} \\
& \bigcup_{K \subseteq\{1, \ldots, d\}, 2 \leq|K| \in\{d, d-1\}} \mathcal{C}_{K-\mathrm{CM}} \quad \subseteq_{\mathrm{III}} \quad \bigcup_{K \subseteq\{1, \ldots, d\}, 2 \leq|K| \leq d} \mathcal{C}_{K-\mathrm{CM}}
\end{aligned}
$$

Recall that, for $d=2$, all the sets are identical and that, for $d \geq 4$, inclusions $I$, $I I I$ and $I V$ are strict. In the case $d=3$, the above figure reduces to

$$
\bigcup_{K \subseteq\{1, \ldots, d\}, 2 \leq|K| \leq 3} \mathcal{C}_{K-\mathrm{CM}} \subseteq_{\mathrm{I}} \quad m(\mathcal{C}, \preceq) \quad \subseteq_{\mathrm{II}} \quad \mathcal{C}_{\text {Kendall-CM }}
$$

where, due to Example 3.4, at least one of the inclusions is strict.

\section{Continuous and Order Preserving Functionals}

In this section, we study minimal copulas in connection with continuous and concordance order preserving functionals and show that each such optimization problem is minimized by some minimal copula. In particular, we show that any continuous functional that is even strictly concordance order preserving is minimized by minimal copulas only.

A map $\phi: \mathcal{C} \rightarrow \mathbb{R}$ is said to be

- continuous if, for any sequence $\left\{C_{n}\right\}_{n \in \mathbb{N}} \subseteq \mathcal{C}$ and any copula $C \in \mathcal{C}$, uniform convergence $\lim _{n \rightarrow \infty} C_{n}=C$ implies $\lim _{n \rightarrow \infty} \phi\left(C_{n}\right)=\phi(C)$.

- concordance order preserving if the inequality $\phi(C) \leq \phi(D)$ holds for all $C, D \in$ $\mathcal{C}$ satisfying $C \preceq D$.

- strictly concordance order preserving if it is concordance order preserving and the strict inequality $\phi(C)<\phi(D)$ holds for all $C, D \in \mathcal{C}$ satisfying $C \preceq D$ with $C \neq D$.

For a given map $\phi: \mathcal{C} \mapsto \mathbb{R}$ and a subset $\mathcal{D} \subseteq \mathcal{C}$, we define the set $m(\phi, \mathcal{D})$ by letting

$$
m(\phi, \mathcal{D}):=\left\{D \in \mathcal{D}: \phi(D)=\inf _{C \in \mathcal{D}} \phi(C)\right\}
$$

It is well known that every continuous functional $\mathcal{C} \mapsto \mathbb{R}$ is minimized by some copula $C \in \mathcal{C}$; see Proposition B.1:

Proposition 4.1 Consider a non-empty and compact subset $\mathcal{D} \subseteq \mathcal{C}$. Then, for every continuous mapping $\phi: \mathcal{C} \mapsto \mathbb{R}$, the $\operatorname{set} m(\phi, \mathcal{D})$ is non-empty. 
Even though a continuous functional $\mathcal{C} \rightarrow \mathbb{R}$ is minimized by some copula $C \in \mathcal{C}$, the calculation of its minimal value can be quite difficult. For an illustration, let us consider the following quite popular optimization problem:

Example 4.1 The map $\phi: \mathcal{C} \rightarrow \mathbb{R}$ given by

$$
\phi(C):=\int_{\mathbb{I}^{d}} \Pi(\mathbf{u}) \mathrm{d} Q^{C}(\mathbf{u})
$$

is a continuous and strictly concordance order preserving functional, and thus, due to Proposition 4.1, $\phi$ is minimized by some copula $C \in \mathcal{C}$. In [7, Corollary 4.1 and Figure 3.2] the authors have determined the value of $\inf _{C \in \mathcal{C}} \phi(C)$ in arbitrary dimension and, for $d=3$, they have presented a minimal copula minimizing $\phi$.

The following result shows that the set of minimal copulas plays a key role when searching for the minimal value of a continuous and (strictly) concordance order preserving functional. It turns out that, for any continuous and concordance order preserving map $\phi$, the set of minimizers contains at least one minimal copula. It further turns out that any continuous map that is even strictly concordance order preserving is minimized by minimal copulas only.

Theorem 4.1 Let $\phi: \mathcal{C} \mapsto \mathbb{R}$ be a continuous and concordance order preserving map.

(1.1) If $\mathcal{D} \subseteq \mathcal{C}$ is compact, then $m(\phi, \mathcal{D})$ contains at least one minimal copula of $(\mathcal{D}, \preceq)$.

(1.2) In particular, $m(\phi, \mathcal{C})$ contains at least one minimal copula of $(\mathcal{C}, \preceq)$.

(1.3) If $d=2$, then $\{W\}=m(\mathcal{C}, \preceq) \subseteq m(\phi, \mathcal{C})$.

Further assume that $\phi$ is also strictly concordance order preserving.

(2.1) If $\mathcal{D} \subseteq \mathcal{C}$ is compact, then $m(\phi, \mathcal{D})$ is a non-empty set of minimal copulas of $(\mathcal{D}, \preceq)$.

(2.2) In particular, $\emptyset \neq m(\phi, \mathcal{C}) \subseteq m(\mathcal{C}, \preceq)$.

(2.3) If $d=2$, then $\{W\}=m(\phi, \mathcal{C})=m(\mathcal{C}, \preceq)$.

(2.4) If $d \geq 3$, then $m(\phi, \mathcal{C}) \neq m(\mathcal{C}, \preceq)$.

Proof The assertions (1.1) and (2.1) follow from Theorem B.1, and (2.4) is immediate from Example 5.3.

We note that the results (2.1) and (2.2) in Theorem 4.1 can not be extended to concordance order preserving functionals that fail to be strictly concordance order preserving since a copula attaining the minimal value of such a functional may fail to be a minimal copula; see, e.g. Corollary 5.1 together with Example 3.3.

\section{Measures of Concordance}

In the following, we apply the results of the previous sections to measures of concordance. First of all, we show that in the class of all continuous and concordance order preserving measures of concordance Kendall's tau is particular since it is minimized 
by every minimal copula. As a consequence of Theorem 4.1, it further turns out that every continuous and strictly concordance order preserving measure of concordance is minimized by minimal copulas only. Since the latter result is applicable to Spearman's rho we may conclude that every copula minimizing Spearman's rho is also a minimizer of Kendall's tau.

We employ the quite general definition of a measure of concordance proposed in [28]; compare also [29-31]: A map $\phi: \mathcal{C} \rightarrow \mathbb{R}$ is said to be a measure of concordance, if it satisfies the following axioms:

(i) $\phi(M)=1$.

(ii) The identity $\phi(\gamma(C))=\phi(C)$ holds for all $\gamma \in \Gamma^{\pi}$ and all $C \in \mathcal{C}$.

(iii) The identity $\phi(\tau(C))=\phi(C)$ holds for all $C \in \mathcal{C}$.

(iv) The identity $\sum_{\nu \in \Gamma^{\nu}} \phi(v(C))=0$ holds for all $C \in \mathcal{C}$.

For the case $d=2$, this definition is in accordance with [32-35].

We first recall the definition of Kendall's tau and then introduce Spearman's rho.

Example 5.1 (Kendall's tau) The map $\kappa: \mathcal{C} \rightarrow \mathbb{R}$ given by

$$
\kappa(C):=\frac{2^{d}}{2^{d-1}-1}\left(\int_{\mathbb{I}^{d}} C(\mathbf{u}) \mathrm{d} Q^{C}(\mathbf{u})-\frac{1}{2^{d}}\right)
$$

is a continuous and concordance order preserving measure of concordance, and is called Kendall's tau; this definition of Kendall's tau is in accordance with that in [27]. $\kappa$ satisfies $\min _{C \in \mathcal{C}} \kappa(C)=-1 /\left(2^{d-1}-1\right)$ and its minimum value is attained by the minimal copula $v_{1}(M)$; see [36, Theorem 5.1] and [5, Remark 3.1 (1)]. It follows from Example 5.4 that Kendall's tau is not strictly concordance order preserving.

Example 5.2 (Spearman's rho) The map $\rho: \mathcal{C} \rightarrow \mathbb{R}$ given by

$$
\rho(C):=\frac{2^{d}(d+1)}{2^{d}-(d+1)}\left(\int_{\mathbb{I}^{d}} \frac{C(\mathbf{u})+(\tau(C))(\mathbf{u})}{2} \mathrm{~d} Q^{\Pi}(\mathbf{u})-\frac{1}{2^{d}}\right)
$$

is a continuous and strictly concordance order preserving measure of concordance; the definition of Spearman's rho used here is in accordance with that in [27]. Even though $\rho$ can be minimized only by minimal copulas (Theorem 4.1), its minimal value is known only for $d \in\{2,3\}$ : In the case $d=2, W$ is the only copula minimizing Spearman's rho with $\rho(W)=-1$ and, for $d=3$, Nelsen and Úbeda-Flores [25, Theorem 4] have shown that $\min _{C \in \mathcal{C}} \rho(C)=-1 / 2$ and that this minimal value is attained only by those minimal copulas satisfying $Q\left[\left\{\mathbf{u} \in \mathbb{I}^{3}: \sum_{i=1}^{3} u_{i}=3 / 2\right\}\right]=1$.

It immediately follows from Theorem 4.1 and Theorem 3.1 that every continuous and strictly concordance order preserving measure of concordance is minimized by minimal copulas only, and that every minimal copula minimizes Kendall's tau:

Corollary 5.1 (1) The inclusions $m(\phi, \mathcal{C}) \subseteq m(\mathcal{C}, \preceq) \subseteq m(\kappa, \mathcal{C})$ hold for every continuous and strictly concordance order preserving measure of concordance $\phi$. 
(2) If $d=2$, then the identities $m(\phi, \mathcal{C})=m(\mathcal{C}, \preceq)=m(\kappa, \mathcal{C})$ hold for every continuous and strictly concordance order preserving measure of concordance $\phi$.

It follows from Example 3.3 that the second inclusion in Corollary 5.1 (1) is strict whenever $d \geq 4$. The next example shows that the first inclusion in Corollary 5.1 (1) is strict whenever $d \geq 3$ :

Example 5.3 For every $d \geq 3$, there exist minimal copulas $C, D \in m(\mathcal{C}, \preceq)$ satisfying $\rho(C)<\rho(D)$. In particular, $m(\rho, \mathcal{C}) \neq m(\mathcal{C}, \preceq)$.

Indeed, for $d=3$, consider the minimal copula $v_{1}(M)$ and the minimal copula $C$ discussed in Example 3.1 (3). Then, by [25, Example 7], we obtain $\rho(C)=-1 / 2<$ $-1 / 3=\rho\left(v_{1}(M)\right)$. For $d \geq 4$, the minimal copulas $v_{1}(M)$ and $v_{1,2}(M)$ satisfy

$$
\rho\left(v_{1,2}(M)\right)=\frac{2^{d+1}-(d-1) d(d+1)}{(d-1) d\left(2^{d}-(d+1)\right)}<\frac{2^{d}-d(d+1)}{d\left(2^{d}-(d+1)\right)}=\rho\left(v_{1}(M)\right)
$$

This proves the assertion.

The relationship between measures of concordance, in particular between bivariate Kendall's tau and bivariate Spearman's rho, has received considerable attention in literature; see, e.g. [37-40]. We are able to contribute to this topic by showing that every minimizer of Spearman's rho is also a minimizer of Kendall's tau:

Corollary 5.2 We have $m(\rho, \mathcal{C}) \subseteq m(\kappa, \mathcal{C})$. Moreover, $m(\rho, \mathcal{C})=m(\kappa, \mathcal{C})$ if and only if $d=2$.

For $d \in\{2,3\}$, the results in Corollary 5.2 are in accordance with Examples 5.1 and 5.2.

We conclude this section by showing that Kendall's tau is not strictly concordance preserving:

Example 5.4 Consider the copula $A: \mathbb{I}^{2} \rightarrow \mathbb{I}$

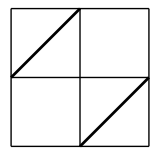

defined as the shuffle of $M$ with respect to the shuffling structure $\left\{\left[\mathbf{a}_{i}, \mathbf{b}_{i}\right]\right\}_{i \in\{1,2\}}$ with

$$
\begin{array}{ll}
\mathbf{a}_{1}=(0,1 / 2) & \mathbf{b}_{1}=(1 / 2,1) \\
\mathbf{a}_{2}=(1 / 2,0) & \mathbf{b}_{2}=(1,1 / 2)
\end{array}
$$

and the copula $B: \mathbb{I}^{2} \rightarrow \mathbb{I}$

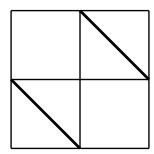


defined as the shuffle of $W$ with respect to the shuffling structure $\left\{\left[\mathbf{a}_{i}, \mathbf{b}_{i}\right]\right\}_{i \in\{1,2\}}$ with

$$
\begin{aligned}
& \mathbf{a}_{1}=(0,0) \quad \mathbf{b}_{1}=(1 / 2,1 / 2) \\
& \mathbf{a}_{2}=(1 / 2,1 / 2) \quad \mathbf{b}_{2}=(1,1)
\end{aligned}
$$

For more details on shuffles of copulas we refer to [41]. The copulas $A$ and $B$ satisfy $A(\mathbf{u}) \leq B(\mathbf{u})$ for all $\mathbf{u} \in \mathbb{I}^{2}$ and hence $A \preceq B$ with $A \neq B$ and, by [5, Corollary 5.2], we obtain $\kappa(A)=\kappa(B)$.

Moreover, for $d \geq 3$, define the functions $C, D: \mathbb{I}^{2} \times \mathbb{I}^{d-2} \rightarrow \mathbb{I}$ by letting $C(\mathbf{u}, \mathbf{v}):=$ $A(\mathbf{u}) \prod_{i=1}^{d-2} v_{i}$ and $D(\mathbf{u}, \mathbf{v}):=B(\mathbf{u}) \prod_{i=1}^{d-2} v_{i}$. By [26, Theorem 6.6.3], $C$ and $D$ are copulas, $C \preceq D$ with $C \neq D$, and, by [5, Corollary 5.2], we obtain $\kappa(C)=\kappa(D)$. Thus, Kendall's tau is not strictly concordance order preserving.

Note that, for $d \geq 4$, the non-strictness of Kendall's tau also follows from Theorem 4.1 and Example 3.3.

Acknowledgements Open access funding provided by Paris Lodron University of Salzburg. Jae Youn Ahn was supported by a National Research Foundation of Korea (NRF) grant funded by the Korean Government (NRF-2017R1D1A1B03032318).

Open Access This article is distributed under the terms of the Creative Commons Attribution 4.0 International License (http://creativecommons.org/licenses/by/4.0/), which permits unrestricted use, distribution, and reproduction in any medium, provided you give appropriate credit to the original author(s) and the source, provide a link to the Creative Commons license, and indicate if changes were made.

\section{Appendices}

\section{Appendix A: Proof of Theorem 3.1}

In this section, we prove Theorem 3.1; the idea of the proof is as follows: For a copula

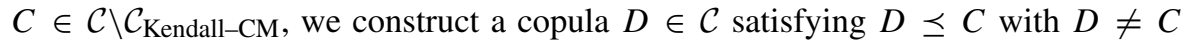
which then implies that $C$ is not a minimal copula. More precisely, we extract some comonotonic part of the given copula $C$ and construct a related copula $D$ in which this comonotonic part is "made non-comonotonic".

The following result provides the basis for the construction of the copula $D$ :

Lemma A.1 For every copula $C \in \mathcal{C} \backslash \mathcal{C}_{\text {Kendall-CM }}$ there exist some $\left.\left.p \in\right] 0,0.5\right]$ and some $\mathbf{a}, \mathbf{b} \in] \mathbf{0}, \mathbf{1}\left[\right.$ with $\mathbf{a} \leq \mathbf{b}$ such that $Q^{C}[[\mathbf{0}, \mathbf{a}]]=p=Q^{C}[[\mathbf{b}, \mathbf{1}]]$.

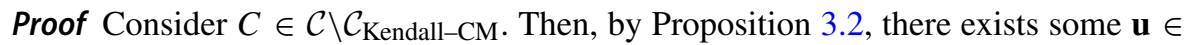
]0, 1 [ satisfying $C(\mathbf{u})>0$ and $Q^{C}[[\mathbf{u}, \mathbf{1}]]>0$. W.l.o.G, let $Q^{C}[[\mathbf{u}, \mathbf{1}]] \leq C(\mathbf{u})$ and put $p:=Q^{C}[[\mathbf{u}, \mathbf{1}]]$. Then $\left.\left.p \in\right] 0,0.5\right]$. Since every copula is continuous, the map $\mathbb{I} \rightarrow \mathbb{I}$ given by $\alpha \mapsto C(\alpha \mathbf{u})$ is continuous as well. Thus, there exists some $\beta \in] 0,1]$ satisfying $\beta \mathbf{u} \in] \mathbf{0}, \mathbf{1}[, \beta \mathbf{u} \leq \mathbf{u}$ and $C(\beta \mathbf{u})=p$. This proves the assertion.

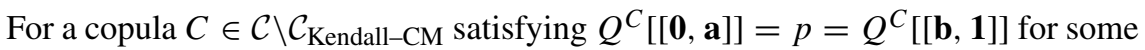
$p \in] 0,0.5]$ and some $\mathbf{a}, \mathbf{b} \in] \mathbf{0}, \mathbf{1}\left[\right.$ with $\mathbf{a} \leq \mathbf{b}$, we first define the maps $C_{(\mathbf{a})}, C_{(\mathbf{b})}$ : $\mathbb{I}^{d} \rightarrow \mathbb{I}$ by letting $C_{(\mathbf{a})}(\mathbf{u}):=\frac{1}{p} Q^{C}[[\mathbf{0}, \mathbf{u}] \cap[\mathbf{0}, \mathbf{a}]]$ and $C_{(\mathbf{b})}(\mathbf{u}):=\frac{1}{p} Q^{C}[[\mathbf{0}, \mathbf{u}] \cap$ 
$[\mathbf{b}, \mathbf{1}]]$. Then $C_{(\mathbf{a})}$ and $C_{(\mathbf{b})}$ are $d$-dimensional distribution functions on $\mathbb{I}^{d}$. We further define the maps $C_{(1, \mathbf{a}, \mathbf{b})}, C_{(2, \mathbf{a}, \mathbf{b})}: \mathbb{I}^{d} \rightarrow \mathbb{I}$ by letting $C_{(1, \mathbf{a}, \mathbf{b})}:=\frac{1}{2} C_{(\mathbf{a})}+\frac{1}{2} C_{(\mathbf{b})}$ and

$$
\begin{aligned}
& C_{(2, \mathbf{a}, \mathbf{b})}(\mathbf{u}) \\
&:= \frac{1}{2} C_{(\mathbf{a})}\left(\eta_{1}(\mathbf{1}, \mathbf{u})\right) \cdot C_{(\mathbf{b})}\left(\eta_{1}(\mathbf{u}, \mathbf{1})\right)+\frac{1}{2} C_{(\mathbf{b})}\left(\eta_{1}(\mathbf{1}, \mathbf{u})\right) \cdot C_{(\mathbf{a})}\left(\boldsymbol{\eta}_{1}(\mathbf{u}, \mathbf{1})\right) \\
&= \frac{1}{2}\left(\frac{1}{p} Q^{C}\left[\left[0, u_{1}\right] \times \mathbb{I}^{d-1} \cap[\mathbf{0}, \mathbf{a}]\right] \cdot \frac{1}{p} Q^{C}\left[\mathbb{I} \times\left[0, u_{2}\right] \times \ldots \times\left[0, u_{d}\right] \cap[\mathbf{b}, \mathbf{1}]\right]\right. \\
&\left.+\frac{1}{p} Q^{C}\left[\left[0, u_{1}\right] \times \mathbb{I}^{d-1} \cap[\mathbf{b}, \mathbf{1}]\right] \cdot \frac{1}{p} Q^{C}\left[\mathbb{I} \times\left[0, u_{2}\right] \times \ldots \times\left[0, u_{d}\right] \cap[\mathbf{0}, \mathbf{a}]\right]\right)
\end{aligned}
$$

Then $C_{(1, \mathbf{a}, \mathbf{b})}$ and $C_{(2, \mathbf{a}, \mathbf{b})}$ are also $d$-dimensional distribution functions on $\mathbb{I}^{d}$.

Lemma A.2 (1) The marginal distribution functions of $C_{(1, \mathbf{a}, \mathbf{b})}$ and $C_{(2, \mathbf{a}, \mathbf{b})}$ are identical.

(2) $C-2 p C_{(1, \mathbf{a}, \mathbf{b})}+2 p C_{(2, \mathbf{a}, \mathbf{b})} \in \mathcal{C}$.

Proof Assertion (1) is immediate from the definition and implies that the map $C$ $2 p C_{(1, \mathbf{a}, \mathbf{b})}+2 p C_{(2, \mathbf{a}, \mathbf{b})}$ has uniform margins. Now, we prove (2). To this end, consider $\mathbf{u}, \mathbf{v} \in \mathbb{I}^{d}$ with $\mathbf{u} \leq \mathbf{v}$. Since $C$ is a copula and $\mathbf{a} \leq \mathbf{b}$, we first obtain

$$
\begin{aligned}
& \sum_{K \subseteq\{1, \ldots, d\}}(-1)^{d-|K|}\left(C-2 p C_{(1, \mathbf{a}, \mathbf{b})}\right)\left(\eta_{K}(\mathbf{u}, \mathbf{v})\right) \\
& \quad=Q^{C}\left[[\mathbf{u}, \mathbf{v}] \cap \mathbb{I}^{d} \backslash([\mathbf{0}, \mathbf{a}] \cup[\mathbf{b}, \mathbf{1}])\right] \geq 0
\end{aligned}
$$

and hence

$$
\begin{aligned}
& \sum_{K \subseteq\{1, \ldots, d\}}(-1)^{d-|K|}\left(C-2 p C_{(1, \mathbf{a}, \mathbf{b})}+2 p C_{(2, \mathbf{a}, \mathbf{b})}\right)\left(\eta_{K}(\mathbf{u}, \mathbf{v})\right) \\
& \quad \geq 2 p \sum_{K \subseteq\{1, \ldots, d\}}(-1)^{d-|K|}\left(C_{(2, \mathbf{a}, \mathbf{b})}\right)\left(\eta_{K}(\mathbf{u}, \mathbf{v})\right) \\
& \quad \geq 0
\end{aligned}
$$

where the last inequality follows from the fact that $C_{(2, \mathbf{a}, \mathbf{b})}$ is a distribution function. It remains to show that the identity $\left(C-2 p C_{(1, \mathbf{a}, \mathbf{b})}+2 p C_{(2, \mathbf{a}, \mathbf{b})}\right)\left(\boldsymbol{\eta}_{k}(\mathbf{u}, \mathbf{0})\right)=0$ holds for every $k \in\{1, \ldots, d\}$ and every $\mathbf{u} \in \mathbb{I}^{d}$, but this follows from the fact $C$ is a copula and $C_{(1, \mathbf{a}, \mathbf{b})}$ and $C_{(2, \mathbf{a}, \mathbf{b})}$ are distribution functions on $\mathbb{I}^{d}$.

Motivated by Lemma A.2, we now put $D:=C-2 p C_{(1, \mathbf{a}, \mathbf{b})}+2 p C_{(2, \mathbf{a}, \mathbf{b})}$ and show that $D \preceq C$ with $D \neq C$.

Lemma A.3 (1) The inequalities $C_{(2, \mathbf{a}, \mathbf{b})}(\mathbf{u}) \leq C_{(1, \mathbf{a}, \mathbf{b})}(\mathbf{u})$ and $D(\mathbf{u}) \leq C(\mathbf{u})$ hold for every $\mathbf{u} \in \mathbb{I}^{d}$.

(2) There exists some $\mathbf{u} \in \mathbb{I}^{d}$ satisfying $C_{(2, \mathbf{a}, \mathbf{b})}(\mathbf{u})<C_{(1, \mathbf{a}, \mathbf{b})}(\mathbf{u})$ and hence $D(\mathbf{u})<$ $C(\mathbf{u})$. 
(3) The inequalities $(\tau(C))_{(2, \mathbf{1}-\mathbf{b}, \mathbf{1}-\mathbf{a})}(\mathbf{u}) \leq(\tau(C))_{(1, \mathbf{1}-\mathbf{b}, \mathbf{1}-\mathbf{a})}(\mathbf{u})$ and $(\tau(D))(\mathbf{u}) \leq$ $(\tau(C))(\mathbf{u})$ hold for every $\mathbf{u} \in \mathbb{I}^{d}$.

(4) We have $D \preceq C$ with $D \neq C$.

Proof To ease notation, for $\mathbf{v} \in \mathbb{I}^{d}$, we put $\mathbf{v}_{1}:=\left(v_{2}, \ldots, v_{d}\right)$. We first prove (1) and consider four cases.

- First, assume that $u_{1}<b_{1}$. We then have $C_{(1, \mathbf{a}, \mathbf{b})}(\mathbf{u})=\frac{1}{2 p} C(\mathbf{u} \wedge \mathbf{a})$ and $C_{(2, \mathbf{a}, \mathbf{b})}(\mathbf{u})=\frac{1}{2 p^{2}} C\left(u_{1} \wedge a_{1}, \mathbf{a}_{1}\right) \cdot Q^{C}\left[\left[b_{1}, 1\right] \times\left[\mathbf{b}_{1}, \mathbf{u}_{1}\right]\right]$.

- If $u_{i}<b_{i}$ for some $i \in\{2, \ldots, d\}$, then we obtain

$$
C_{(2, \mathbf{a}, \mathbf{b})}(\mathbf{u})=\frac{1}{2 p^{2}} C\left(u_{1} \wedge a_{1}, \mathbf{a}_{1}\right) \cdot Q^{C}\left[\left[b_{1}, 1\right] \times\left[\mathbf{b}_{1}, \mathbf{u}_{1}\right]\right]=0 \leq C_{(1, \mathbf{a}, \mathbf{b})}(\mathbf{u})
$$

- If $u_{i} \geq b_{i} \geq a_{i}$ for every $i \in\{2, \ldots, d\}$, then we obtain

$$
\begin{aligned}
C_{(2, \mathbf{a}, \mathbf{b})}(\mathbf{u}) & =\frac{1}{2 p^{2}} C\left(u_{1} \wedge a_{1}, \mathbf{a}_{1}\right) \cdot Q^{C}\left[\left[b_{1}, 1\right] \times\left[\mathbf{b}_{1}, \mathbf{u}_{1}\right]\right] \\
& \leq \frac{1}{2 p^{2}} C\left(u_{1} \wedge a_{1}, \mathbf{a}_{1}\right) \cdot Q^{C}[[\mathbf{b}, \mathbf{1}]] \\
& =\frac{1}{2 p} C\left(u_{1} \wedge a_{1}, \mathbf{a}_{1}\right) \\
& =C_{(1, \mathbf{a}, \mathbf{b})}(\mathbf{u})
\end{aligned}
$$

- Now, assume that $u_{1} \geq b_{1} \geq a_{1}$. We then have

$$
\begin{aligned}
C_{(1, \mathbf{a}, \mathbf{b})}(\mathbf{u})= & \frac{1}{2 p}\left(C\left(a_{1}, \mathbf{u}_{1} \wedge \mathbf{a}_{1}\right)+Q^{C}\left[\left[b_{1}, u_{1}\right] \times\left[\mathbf{b}_{1}, \mathbf{u}_{1}\right]\right]\right) \\
C_{(2, \mathbf{a}, \mathbf{b})}(\mathbf{u})= & \frac{1}{2 p^{2}}\left(C(\mathbf{a}) \cdot Q^{C}\left[\left[b_{1}, 1\right] \times\left[\mathbf{b}_{1}, \mathbf{u}_{1}\right]\right]\right. \\
& \left.+Q^{C}\left[\left[b_{1}, u_{1}\right] \times\left[\mathbf{b}_{1}, \mathbf{1}\right]\right] \cdot C\left(a_{1}, \mathbf{u}_{1} \wedge \mathbf{a}_{1}\right)\right)
\end{aligned}
$$

- If $u_{i}<b_{i}$ for some $i \in\{2, \ldots, d\}$, then we obtain

$$
\begin{aligned}
C_{(2, \mathbf{a}, \mathbf{b})}(\mathbf{u}) & =\frac{1}{2 p^{2}} Q^{C}\left[\left[b_{1}, u_{1}\right] \times\left[\mathbf{b}_{1}, \mathbf{1}\right]\right] \cdot C\left(a_{1}, \mathbf{u}_{1} \wedge \mathbf{a}_{1}\right) \\
& \leq \frac{1}{2 p^{2}} Q^{C}[[\mathbf{b}, \mathbf{1}]] \cdot C\left(a_{1}, \mathbf{u}_{1} \wedge \mathbf{a}_{1}\right)=\frac{1}{2 p} C\left(a_{1}, \mathbf{u}_{1} \wedge \mathbf{a}_{1}\right) \\
& =C_{(1, \mathbf{a}, \mathbf{b})}(\mathbf{u})
\end{aligned}
$$

- If $u_{i} \geq b_{i} \geq a_{i}$ for every $i \in\{2, \ldots, d\}$, then we obtain

$$
C_{(2, \mathbf{a}, \mathbf{b})}(\mathbf{u})
$$




$$
\begin{aligned}
& =\frac{1}{2 p^{2}}\left(C(\mathbf{a}) \cdot Q^{C}\left[\left[b_{1}, 1\right] \times\left[\mathbf{b}_{1}, \mathbf{u}_{1}\right]\right]+Q^{C}\left[\left[b_{1}, u_{1}\right] \times\left[\mathbf{b}_{1}, \mathbf{1}\right]\right] \cdot C(\mathbf{a})\right) \\
& =\frac{1}{2 p}\left(Q^{C}\left[\left[b_{1}, 1\right] \times\left[\mathbf{b}_{1}, \mathbf{u}_{1}\right]\right]+Q^{C}\left[\left[b_{1}, u_{1}\right] \times\left[\mathbf{b}_{1}, \mathbf{1}\right]\right]\right) \\
& =\frac{1}{2 p}\left(Q^{C}[[\mathbf{b}, \mathbf{u}]]+Q^{C}\left[\left[u_{1}, 1\right] \times\left[\mathbf{b}_{1}, \mathbf{u}_{1}\right]\right]+Q^{C}\left[\left[b_{1}, u_{1}\right] \times\left[\mathbf{b}_{1}, \mathbf{1}\right]\right]\right) \\
& \leq \frac{1}{2 p}\left(Q^{C}[[\mathbf{b}, \mathbf{u}]]+Q^{C}\left[\left[u_{1}, 1\right] \times\left[\mathbf{b}_{1}, \mathbf{1}\right]\right]+Q^{C}\left[\left[b_{1}, u_{1}\right] \times\left[\mathbf{b}_{1}, \mathbf{1}\right]\right]\right) \\
& =\frac{1}{2 p}\left(Q^{C}[[\mathbf{b}, \mathbf{u}]]+Q^{C}[[\mathbf{b}, \mathbf{1}]]\right) \\
& =\frac{1}{2 p}\left(p+Q^{C}[[\mathbf{b}, \mathbf{u}]]\right) \\
& =\frac{1}{2 p}\left(C(\mathbf{a})+Q^{C}[[\mathbf{b}, \mathbf{u}]]\right) \\
& =C_{(1, \mathbf{a}, \mathbf{b})}(\mathbf{u})
\end{aligned}
$$

This proves (1), and (2) follows from the inequality $C_{(2, \mathbf{a}, \mathbf{b})}(\mathbf{a})=0<1 / 2=$ $C_{(1, \mathbf{a}, \mathbf{b})}(\mathbf{a})$.

We now prove (3). The inequality $(\tau(C))_{(2, \mathbf{1}-\mathbf{b}, \mathbf{1}-\mathbf{a})}(\mathbf{u}) \leq(\tau(C))_{(1, \mathbf{1}-\mathbf{b}, \mathbf{1}-\mathbf{a})}(\mathbf{u})$ for all $\mathbf{u} \in \mathbb{I}^{d}$ is immediate from (1) and the fact that $\mathbf{1}-\mathbf{b} \leq \mathbf{1}-\mathbf{a}$. By [20, Theorem 4.1], we further have

$$
\begin{aligned}
(\tau(D)) & (\mathbf{u}) \\
= & \sum_{L \subseteq\{1, \ldots, d\}}(-1)^{d-|L|} D\left(\boldsymbol{\eta}_{L}(\mathbf{1}-\mathbf{u}, \mathbf{1})\right) \\
= & (\tau(C))(\mathbf{u})+2 p \sum_{L \subseteq\{1, \ldots, d\}}(-1)^{d-|L|}\left(C_{(2, \mathbf{a}, \mathbf{b})}\left(\eta_{L}(\mathbf{1}-\mathbf{u}, \mathbf{1})\right)\right. \\
& \left.-C_{(1, \mathbf{a}, \mathbf{b})}\left(\eta_{L}(\mathbf{1}-\mathbf{u}, \mathbf{1})\right)\right)
\end{aligned}
$$

for all $\mathbf{u} \in \mathbb{I}^{d}$. Moreover, applying [14, Theorem 2.2], the identities

$$
\begin{aligned}
2 p & \sum_{L \subseteq\{1, \ldots, d\}}(-1)^{d-|L|} C_{(1, \mathbf{a}, \mathbf{b})}\left(\boldsymbol{\eta}_{L}(\mathbf{1}-\mathbf{u}, \mathbf{1})\right) \\
& =Q^{C}[[\mathbf{1}-\mathbf{u}, \mathbf{1}] \cap[\mathbf{0}, \mathbf{a}]]+Q^{C}[[\mathbf{1}-\mathbf{u}, \mathbf{1}] \cap[\mathbf{b}, \mathbf{1}]] \\
& =Q^{\tau(C)}[[\mathbf{0}, \mathbf{u}] \cap[\mathbf{1}-\mathbf{a}, \mathbf{1}]]+Q^{\tau(C)}[[\mathbf{0}, \mathbf{u}] \cap[\mathbf{0}, \mathbf{1}-\mathbf{b}]] \\
& =2 p(\tau(C))_{(1, \mathbf{1}-\mathbf{b}, \mathbf{1}-\mathbf{a})(\mathbf{u})}
\end{aligned}
$$

and

$$
2 p \sum_{L \subseteq\{1, \ldots, d\}}(-1)^{d-|L|} C_{(2, \mathbf{a}, \mathbf{b})}\left(\eta_{L}(\mathbf{1}-\mathbf{u}, \mathbf{1})\right)
$$




$$
\begin{aligned}
= & \frac{1}{p} Q^{C}\left[\left[1-u_{1}, 1\right] \times \mathbb{I}^{d-1} \cap[\mathbf{0}, \mathbf{a}]\right] \cdot Q^{C}\left[\mathbb{I} \times\left[\mathbf{1}-\mathbf{u}_{1}, \mathbf{1}\right] \cap[\mathbf{b}, \mathbf{1}]\right] \\
& +\frac{1}{p} Q^{C}\left[\left[1-u_{1}, 1\right] \times \mathbb{I}^{d-1} \cap[\mathbf{b}, \mathbf{1}]\right] \cdot Q^{C}\left[\mathbb{I} \times\left[\mathbf{1}-\mathbf{u}_{1}, \mathbf{1}\right] \cap[\mathbf{0}, \mathbf{a}]\right] \\
= & \frac{1}{p} Q^{\tau(C)}\left[\left[0, u_{1}\right] \times \mathbb{I}^{d-1} \cap[\mathbf{1}-\mathbf{a}, \mathbf{1}]\right] \cdot Q^{\tau(C)}\left[\mathbb{I} \times\left[\mathbf{0}, \mathbf{u}_{1}\right] \cap[\mathbf{0}, \mathbf{1}-\mathbf{b}]\right] \\
& +\frac{1}{p} Q^{\tau(C)}\left[\left[0, u_{1}\right] \times \mathbb{I}^{d-1} \cap[\mathbf{0}, \mathbf{1}-\mathbf{b}]\right] \cdot Q^{\tau(C)}\left[\mathbb{I} \times\left[\mathbf{0}, \mathbf{u}_{1}\right] \cap[\mathbf{1}-\mathbf{a}, \mathbf{1}]\right] \\
= & 2 p(\tau(C))_{(2, \mathbf{1}-\mathbf{b}, \mathbf{1}-\mathbf{a})}(\mathbf{u})
\end{aligned}
$$

hold for all $\mathbf{u} \in \mathbb{I}^{d}$ and hence

$$
\begin{aligned}
(\tau(D))(\mathbf{u}) & =(\tau(C))(\mathbf{u})+2 p\left((\tau(C))_{(2, \mathbf{1}-\mathbf{b}, \mathbf{1}-\mathbf{a})}(\mathbf{u})-(\tau(C))_{(1, \mathbf{1}-\mathbf{b}, \mathbf{1}-\mathbf{a})}(\mathbf{u})\right) \\
& \leq(\tau(C))(\mathbf{u})
\end{aligned}
$$

for all $\mathbf{u} \in \mathbb{I}^{d}$. This proves (3), and (4) is a consequence of (1), (2) and (3).

\section{Appendix B: Some Topological Results}

In this section, we recall some useful topological results. We start with the extreme value theorem:

Proposition B.1 Consider a real-valued and continuous mapping $\phi$ on a non-empty and compact topological space $X$. Then there exists some $y \in X$ satisfying $\phi(y)=$ $\inf _{x \in X} \phi(x)$.

The next result is immediate from [42, Proposition 1] and [43, Theorem 1]:

Proposition B.2 Consider a non-empty and compact topological space X equipped with a closed order $\lessdot$. Then, for every $x \in X$, there exists some minimal element $y \in X$ satisfying $y \lessdot x$.

Applying the above propositions, we obtain the following result:

Theorem B.1 Consider a non-empty and compact topological space X equipped with a closed order $\lessdot$ and let $\phi$ be a real-valued and continuous mapping on $X$.

(1) If $\phi$ is monotone, then the set of all minimizers of $\phi$ is non-empty and contains at least one minimal element of $X$.

(2) If $\phi$ is strictly monotone, then every minimizer of $\phi$ is a minimal element of $X$.

Proof Assertion (1) is immediate from Propositions B.1 and B.2. We now prove (2). To this end, consider any $y \in X$ satisfying $\phi(y)=\inf _{x \in X} \phi(x)$ and any $z \in X$ satisfying $z \lessdot y$. Since $\phi$ is monotone, we obtain $\phi(z)=\phi(y)$, and now the strict monotonicity of $\phi$ yields $z=y$. Therefore, $y$ is a minimal element of $X$. This proves (2) and hence the assertion. 


\section{References}

1. Durante, F., Sempi, C.: Principles of Copula Theory. CRC Press, Boca Raton (2016)

2. Dhaene, J., Denuit, M., Goovaerts, M.J., Kaas, R., Vyncke, D.: The concept of comonotonicity in actuarial science and finance: theory. Insur. Math. Econ. 31(1), 3-33 (2002)

3. Cheung, K.C., Lo, A.: Characterizing mutual exclusivity as the strongest negative multivariate dependence structure. Insur. Math. Econ. 55, 180-190 (2014)

4. Dhaene, J., Denuit, M.: The safest dependence structure among risks. Insur. Math. Econ. 25(1), 11-21 (1999)

5. Fuchs, S., McCord, Y., Schmidt, K.D.: Characterizations of copulas attaining the bounds of multivariate Kendall's tau. J. Optim. Theory Appl. 178(2), 424-438 (2018)

6. Lee, W., Ahn, J.Y.: On the multidimensional extension of countermonotonicity and its applications. Insur. Math. Econ. 56, 68-79 (2014)

7. Wang, B., Wang, R.: The complete mixability and convex minimization problems with monotone marginal densities. J. Multivar. Anal. 102(10), 1344-1360 (2011)

8. Wang, B., Wang, R.: Joint mixability. Math. Oper. Res. 41(3), 808-826 (2016)

9. Dhaene, J., Linders, D., Schoutens, W., Vyncke, D.: A multivariate dependence measure for aggregating risks. J. Comput. Appl. Math. 263, 78-87 (2014)

10. Ahn, J.Y.: Negative dependence concept in copulas and the marginal free herd behavior index. J. Comput. Appl. Math. 288, 304-322 (2015)

11. Lee, W., Cheung, K.C., Ahn, J.Y.: Multivariate countermonotonicity and the minimal copulas. J. Comput. Appl. Math. 317, 589-602 (2017)

12. Rüschendorf, L., Uckelmann, L.: Variance minimization and random variables with constant sum. In: Cuadras, C.M., Fortiana, J., Rodriguez-Lallena, J.A. (eds.) Distributions with Given Marginals and Statistical Modelling, pp. 211-222. Kluwer, Dordrecht (2002)

13. Genest, C., Nešlehová, J., Ben Ghorbal, N.: Estimators based on Kendall's tau in multivariate copula models. Aust. N. Z. J. Stat. 53, 157-177 (2011)

14. Fuchs, S.: A biconvex form for copulas. Depend. Model. 4, 63-75 (2016)

15. Lux, T., Papapantoleon, A.: Improved Fréchet-Hoeffding bounds on $d$-copulas and applications in model-free finance. Ann. Appl. Probab. 27(6), 3633-3671 (2017)

16. Preischl, M.: Bounds on integrals with respect to multivariate copulas. Depend. Model. 4, 277-287 (2016)

17. Puccetti, G., Wang, R.: Extremal dependence concepts. Stat. Sci. 30(4), 485-517 (2015)

18. Tankov, P.: Improved Fréchet bounds and model-free pricing of multi-asset options. J. Appl. Probab. 48(2), 389-403 (2011)

19. Nelsen, R.B.: An Introduction to Copulas, 2nd edn. Springer, New York (2006)

20. Fuchs, S.: Multivariate copulas: Transformations, symmetry, order and measures of concordance. Kybernetika 50(5), 725-743 (2014)

21. Joe, H.: Multivariate concordance. J. Multivar. Anal. 35, 12-30 (1990)

22. Müller, A., Scarsini, M.: Some remarks on the supermodular order. J. Multivar. Anal. 73, 107-119 (2000)

23. Müller, A., Scarsini, M.: Stochastic order relations and lattices of probability measures. SIAM J. Optim. 16(4), 1024-1043 (2006)

24. Fuchs, S.: Conditioning of copulas: Transformations, invariance and measures of concordance. J. Math. Anal. Appl. 462(1), 521-541 (2018)

25. Nelsen, R.B., Úbeda-Flores, M.: Directional dependence in multivariate distributions. Ann. Inst. Stat. Math. 64, 677-685 (2012)

26. Schweizer, B., Sklar, A.: Probabilistic Metric Spaces. North-Holland, New York (1983)

27. Nelsen, R.B.: Concordance and copulas: a survey. In: Cuadras, C.M., Fortiana, J., Rodriguez-Lallena, J.A. (eds.) Distributions with Given Marginals and Statistical Modelling, pp. 169-177. Kluwer, Dordrecht (2002)

28. Fuchs, S.: Copula-induced measures of concordance. Depend. Model. 4, 205-214 (2016)

29. Dolati, A., Úbeda-Flores, M.: On measures of multivariate concordance. J. Probab. Stat. Sci. 4(2), 147-163 (2006)

30. Taylor, M.D.: Multivariate measures of concordance. Ann. Inst. Stat. Math. 59, 789-806 (2007)

31. Taylor, M.D.: Multivariate measures of concordance for copulas and their marginals. Depend. Model. 4, 224-236 (2016) 
32. Edwards, H.H., Mikusiński, P., Taylor, M.D.: Measures of concordance determined by $D_{4}$-invariant copulas. Int. J. Math. Math. Sci. 2004(70), 3867-3875 (2004)

33. Edwards, H.H., Mikusiński, P., Taylor, M.D.: Measures of concordance determined by $D_{4}$-invariant measures on $(0,1)^{2}$. Proc. Am. Math. Soc. 133(5), 1505-1513 (2005)

34. Fuchs, S., Schmidt, K.D.: Bivariate copulas: Transformations, asymmetry and measures of concordance. Kybernetika 50(1), 109-125 (2014)

35. Scarsini, M.: On measures of concordance. Stochastica 8(3), 201-218 (1984)

36. Úbeda-Flores, M.: Multivariate versions of Blomqvist's beta and Spearman's footrule. Ann. Inst. Stat. Math. 57, 781-788 (2005)

37. Capéraà, P., Genest, C.: Spearman's $\rho$ is larger than Kendall's $\tau$ for positively dependent random variables. J. Nonparametr. Stat. 2(2), 183-194 (1993)

38. Fredricks, G.A., Nelsen, R.B.: On the relationship between Spearman's rho and Kendall's tau for pairs of continuous random variables. J. Stat. Plann. Inference 137(7), 2143-2150 (2007)

39. Hürlimann, W.: Hutchinson-Lai's conjecture for bivariate extreme value copulas. Stat. Probab. Lett. 61(2), 191-198 (2003)

40. Schreyer, M., Paulin, R., Trutschnig, W.: On the exact region determined by Kendall's $\tau$ and Spearman's $\rho$. J. R. Stat. Soc. Ser. B. Stat. Methodol. 79, 613-633 (2017)

41. Durante, F., Fernández-Sánchez, J.: Multivariate shuffles and approximation of copulas. Stat. Probab. Lett. 80, 1827-1834 (2010)

42. Nachbin, L.: Topology and Order. D. van Nostrand, Princeton (1965)

43. Ward, L.E.: Partially ordered topological spaces. Proc. Am. Math. Soc. 5, 144-161 (1954)

Publisher's Note Springer Nature remains neutral with regard to jurisdictional claims in published maps and institutional affiliations. 